Elisabeth Knipf-Komlósi*

Márta Müller**

ELTE Budapest
UDK 811.112.2:323.15(439=112.2)

DOI: $10.4312 /$ linguistica.60.2.65-78

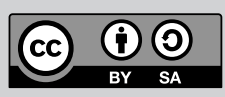

\title{
AKTUELLE FRAGEN DER DEUTSCHEN MINDERHEITENFORSCHUNG IN UNGARN
}

\section{EINLEITUNG}

In Ungarn gilt die deutsche Minderheit - neben zwölf anderen staatlich anerkannten Minderheiten - als die zweitgrößte nationale Minderheit nach der ethnischen Minderheit der Roma. Der Assimilationsprozess erstreckt sich auf die lange Zeit ihres Daseins in Ungarn, doch wurde dieser durch die bewegte Geschichte des 20. Jahrhunderts erheblich beschleunigt, trotz der Tatsache, dass der Schutz und die Rechte der Minderheiten zum Erhalt und zur Pflege ihrer Muttersprache in der Gegenwart gesetzlich gesichert sind. Dies ist nicht nur in der Verfassung Ungarns, sondern auch auf der Verwaltungsebene, durch die vor Ort tätigen lokalen Minderheitenselbstverwaltungen garantiert.

Es erfordert weitere Untersuchungen, um die Frage zu beantworten, ob und wie der gegebene gesetzliche Rahmen von den einzelnen Minderheiten genutzt wird. Dies hängt nicht nur von sprachlichen Aspekten der Minderheitengemeinschaft ab, sondern in großem Maße auch von externen Faktoren, wie von der Selbstinitiative und Aktivität der Minderheit, auf welche Weise die einzelnen Minderheiten für den Erhalt ihrer Sprache und Kultur, für das Fortbestehen ihrer Sprachgemeinschaft etwas unternehmen, ob dazu Strategien vorhanden sind. Aufgrund der bisherigen Ergebnisse der Minderheitenforschung in Ungarn sowie aufgrund der gut organisierten Verwaltungsebenen, der aktiven Gremien und Körperschaften, kann behauptet werden, dass die Ungarndeutschen zielbewusst vieles zum Erhalt ihrer Minderheit unternehmen. Die deutsche Sprache und Kultur sowie deren Erhalt spielte im Leben der Deutschen in Ungarn über all die soziohistorischen Umwälzungen der Jahrhunderte hinweg eine zentrale Rolle. Die zur Förderung des Erhalts der Sprache und Kultur eingesetzten Maßnahmen und Initiativen werden sowohl seitens des deutschsprachigen Sprachraumes als auch von Seiten der Minderheit selbst durch wissenschaftliche Forschungen auf universitärer Ebene unterstützt.

Vor diesem Hintergrund werden im vorliegenden Beitrag nach einer kurzen Einleitung in den aktuellen Kontext des Minderheitenbegriffs zwei ausgewählte

\footnotetext{
knipfe@freemail.hu

** muller.marta@btk.elte.hu
} 
Forschungsfragen zu laufenden Projekten in Ungarn ${ }^{1}$, zu zweisprachigen kommunikativen Praktiken und zu Sprachbiografien vorgestellt.

\title{
2 DER AKTUELLE KONTEXT DES MINDERHEITENBEGRIFFS
}

In den letzten zwei Jahrzehnten entstand eine aus forschungstheoretischer Sicht wichtige Debatte um die Definition des Sprachinselbegriffs, auf den hier kurz eingegangen werden soll. Für die Experten dieses Bereichs ist es eindeutig, dass der bislang in der Fachliteratur eingebürgerte Begriff 'Sprachinsel' unter den gegenwärtigen Umständen der Globalisierung, der weltweiten Mobilität und Internationalisierung wohl kaum noch gebraucht werden kann, zumal die Metapher 'Insel' für diese Erscheinung in der Gegenwart nicht mehr zutrifft. Tatsache ist auch, dass der Begriff seit Anfang der Sprachinselforschung an relevanten Merkmalen eingebüßt hatte, die in der frühen Phase als salient und forschungshistorisch als wichtig erachtet wurden, wie die Arealität, die Territorialität mit dem punktuellen, verstreuten Auftreten, die räumliche $\mathrm{Ab}$ grenzung von der Umgebung, eine sprachlich-kulturelle Isoliertheit, eine sprachlich differente Mehrheitsgesellschaft sowie ausgeprägte ethnisch-kulturelle Differenzen der Minderheiten. Es geht um Merkmale, die in den gegenwärtigen Konstellationen deutscher Minderheiten wohl nirgends auf der Welt mehr zutreffen, denn spätestens ab der zweiten Hälfte des 20. Jahrhunderts haben fast alle diese Faktoren an Bedeutung verloren. So rückte in der einschlägigen Forschungsliteratur anstelle des 'Sprachinsel'Begriffs allmählich der Begriff 'Sprachminderheit' in den Vordergrund, dessen Extension umfassender ist und auf die gegenwärtigen Sprachkonstellationen von deutschen Minderheiten in der Welt mehr zutrifft:

\begin{abstract}
Man kann unter soziolinguistischem Blickwinkel nicht davon absehen, dass das Deutsche nicht einfach eine Klasse von historisch miteinander verwandten Varietäten ist. Vielmehr ist das Deutsche eine entwickelte westeuropäische Standardsprache mit einer voll durchgeführten funktionalen Differenzierung, die dem Sprachinselidiom gerade abgeht (Eichinger 1997: 157).
\end{abstract}

Obgleich der Begriff Minderheitensprache in Fachkreisen bekannt und auch in Gebrauch ist, sind damit immer noch begrifflich-definitorische Unsicherheiten verbun$\mathrm{den}^{2}$. In modernen Gesellschaften werden Minderheitensprachen und ihre Sprecherinnen und Sprecher als ethnisch differente Kulturträgerinnen und -träger betrachtet, die

1 Die Projekte zur Erforschung der Einstellungen zu den Varietäten des Deutschen sowie zu den sprachbiografischen Interviews der Ungarndeutschen sind am Germanistischen Institut der Eötvös Loránd Universität Budapest angesiedelt.

2 Der Begriff Minderheit hat in den letzteren Jahrzehnten eine beachtliche Definitionsvielfalt und diverse Interpretationen in allen Lebensbereichen erlebt, auf die hier nicht eingegangen werden kann. 
ein eigenes Recht auf ihre Sprache und Kultur haben ${ }^{3}$, wobei diese Rechte häufig als Zankapfel in politischen Kreisen auftauchen.

Als bekannte Merkmale des Deutschen als Minderheitensprache können in den bisherigen Forschungen folgende erwähnt werden:

- die Überdachung der Minderheitensprache durch die jeweilige Landessprache als Mehrheitssprache, d. h. Varietäten der Minderheitensprache werden zugunsten der Landessprache aufgegeben,

- die Heterogenität der Sprachlagen der deutschen Sprache (teils innere Mehrsprachigkeit bei Minderheitensprecherinnen und -sprechern z. B. nur rezeptive Verstehenskompetenz des deutschen Dialekts, gesteuert erlernter deutscher Standard bei der jüngeren Generation),

- Alternation und Mischung der Sprachen und Varietäten (Landes- und Minderheitensprache) in ein und derselben Kommunikationssituation unter Minderheiten,

- der stark eingeschränkte Kommunikationsraum der Minderheitensprecherinnen und -sprecher, der sich in der Gegenwart nur noch sporadisch auf den unmittelbaren Nähebereich beschränkt (Familie, unmittelbarer Freundes- und Verwandtenkreis evtl. doch eher selten unter mundartkundigen Sprecherinnen und Sprechern in der Ortsgemeinschaft).

Im Vergleich zu jener deutschen Sprache, die von den jüngeren Generationen dieser Minderheit in den letzten vier bis fünf Jahrzehnten durch einen gesteuerten Deutschunterricht erlernt wurde und wird, ist diese Varietät des Deutschen aus dialektologischer und sprachhistorischer Sicht eine eigene Varietät. Vor diesem Hintergrund sind jene aus soziolinguistischer Perspektive wichtigen empirischen Erhebungen, die zahlreichen Interviews und Fragebogenerhebungen mit Sprachminderheitenangehörigen zu erwähnen - durchgeführt von Forschern der Dialektologie und Soziolinguistik in Ungarn, - die unter Beweis stellen, dass Minderheitensprecherinnen und -sprecher zwar ihre deutsche Varietät (den lokalen Dialekt) als wichtiges Kommunikationsmittel im Laufe der letzten vier bis fünf Jahrzehnte aufgegeben haben, doch das Bewusstsein ihrer Zugehörigkeit zum deutschen Sprachgebiet, zu ihrer Abstammung ${ }^{4}$, noch vorhanden ist. Diese kleinen Signale der Loyalität können in Form eines Familiennamens oder durch weitertradierte deutsche Familienbräuche, evtl. durch die in den Familien noch lebenden Narrativen von deutschen Vorfahren auftauchen. Seit Beginn des neuen

3 Vgl. dazu die Europäische Charta der Regional- und Minderheitensprachen des Europarates, in der Fördermaßnahmen zur Sicherung der traditionellen kulturellen und sprachlichen Mannigfaltigkeit festgelegt sind, vgl. auch Knipf/Müller (2019: 483-500).

4 Der Kontext dieser Ausführungen bezieht sich auf die Region der MOE-Länder, da nämlich in den deutschen Sprachgemeinschaften in Übersee der Gegenwart eine von den mittelosteuropäischen Sprachminderheiten in mehrfacher Hinsicht abweichende Konstellation besteht: Letztere haben nach der Öffnung des Eisernen Vorhangs Ende des 20. Jahrunderts eine viel größere sprachliche und kulturelle Nähe und damit einen intensiveren Einfluss des Deutschen in ihrem Alltag, durch die Medien und Reisen erlebt als die deutschen Sprachminderheiten in Übersee. 
Jahrtausends kann dieses Bewusstsein, nennen wir es Loyalität, aufgrund von persönlichen Erfahrungen, Reisen, Begegnungen der Sprecherinnen und -sprecher z. B. in Mittelost- und Südeuropa, aufgrund der Arbeitsmöglichkeiten im deutschen Sprachgebiet wieder erweckt und gestärkt werden. Wenngleich die Insellage der Sprachminderheiten schon seit langer Zeit im metaphorischen Sinne keine Insellage mehr ist, stellt die deutsche Minderheit in Ost-, Mittelost- und Südosteuropa, aufgrund ihrer Genese und ihrer spezifischen Stellung im deutschen Sprachgebiet, im Gefüge der plurizentrischen deutschen Sprache, heute noch eine unumstrittene eigenständige Sprachminderheit dar, wie dies von einer Sprecherin der älteren Generation ${ }^{5}$ auch formuliert wird:

(1) Wie mir Schwowe haitzutag rete, des vrsteh'n mir am beschte, des is a komisches Deitsch, wal do sain viel ungarische Wertr a schun drin, awer mr vrstehn des halt, des is jetz unser Sproch..."

(Wie wir Schwaben heutzutage reden, das verstehen wir am besten, das ist ein komisches Deutsch, weil da sind schon viele ungarische Wörter dabei, aber wir verstehen das halt, das ist jetzt unsere Sprache.) (Frau, 68 J.)

\section{ZUR NOTWENDIGKEIT DER ERFORSCHUNG DER MINDERHEITENSPRACHE ${ }^{6}$ IN DER GEGENWART}

Forschungen zur deutschen Sprache der Minderheit in Ungarn können - wie im Falle vieler Minderheitensprachen - vor allem durch historisch, politisch und sozial bedingte Ursachen begründet und daraus folgend anhand von soziolinguistischen Untersuchungen durchgeführt werden. Als Ursachen der Notwendigkeit dieser Forschungen sind anzuführen:

1) die rapide schrumpfende Zahl der noch aktiven Sprecherinnen und Sprecher,

2) der in den letzten Jahrzehnten erfolgte Domänen- und Funktionsverlust, d. h. der Verlust der Vitalität der lokalen deutschen Dialekte,

3) der vollzogene Sprachwechsel der Generationen,

4) das seit Jahrzehnten fehlende Weitertradieren der deutschen Sprache (primäre Sprachsozialisation) in den Familien.

5 Unsere Beispiele in diesem Beitrag stammen aus zwei Gebieten der Ungarndeutschen: die ersten drei Sprachbelege stammen aus Südungarn in einer rheinfränkisch-pfälzischen Mundart, die anderen aus dem Dialektgebiet im Ofner Bergland, Budapest-Umgebung (ein bairischer Dialektraum).

6 In einem kurzen Rückblick auf die Vergangenheit sollen - ohne Anspruch auf Vollständigkeit einige oft behandelte Themenbereiche bzgl. der Minderheitensprache seit den 1960er Jahren des 20. Jahrhunderts in Ungarn erwähnt werden: die Beschreibung von Ortsgrammatiken der gesprochenen deutschen Dialekte fokussiert auf den phonetischen, lexikalischen, teils auch syntaktischen Bereich (z. B. Manherz 1986, Wild 2003, Márkus 2014), die Sprachgebrauchsforschungen, die Folgen des voranschreitenden Sprachwechsels, Anfang der 90er Jahre (Erb/Knipf 2000), Forschungen zu Sprachkontakten, Lehnwörtern (Erb 2012), zu Fachsprachen (Müller 2011), zahlreiche Forschungen zur Volkskunde der Ungarndeutschen (Manherz 1986). 
Hierbei spielen auch weitere relevante Faktoren wie soziologische, psycholinguistische etc. eine Rolle, doch diese unterstreichen allesamt die Notwendigkeit der Erforschung der Sprache der Minderheit, vor allem auf der Sprachgebrauchsebene. Selbst Minderheitensprecherinnen und -sprecher geben zu, dass in der Alltagskommunikation oft aus Bequemlichkeitsgründen die geläufigere Sprache, das Ungarische gewählt wird. D. h. dass bestimmte soziopsychische Dispositionen, gesellschaftliche Erwartungen, Attitüden ihre Sprachwahl steuern.

Bisherige Erkenntnisse aus Forschungen deuten auf gravierende Veränderungen in den Sprachenverhältnissen der deutschen Minderheit nicht nur in Ungarn, auch in Mittelosteuropa, hin. Ein kurzer Umriss von soziokulturellen Faktoren dieser Minderheit in einem breiteren Kontext:

- bi- und multikulturelle, in der Gegenwart dominant ein- bzw. landessprachliche Sprachumgebung, deren Einfluss konstant und intensiv ist,

- Verlust des Deutschen als distanzsprachliche Varietät als Verkehrssprache in der Region Mittelosteuropas,

- das Fehlen (z. B. durch Abwanderung) der Trägerschicht einer bildungssprachlichen Varietät, das zu einer Instabilität der Sprachgemeinschaft führen kann,

- die Folge des Domänen- und Funktionsverlustes von Sprachgebrauchssituationen der Minderheitensprache: vermehrt auftretende sprachliche Mischformen, ein mehrsprachiger Sprachmodus, der zu einem allmählichen Sprachabbau (Monolingualismus) bei Minderheitensprecherinnen und -sprechern führt,

- generationenbedingte Sprachmodalitäten, z. B. häufige asymmetrsiche Kommunikationssituationen (zweier Generationen) in zwei Sprachen ohne Verstehensschwierigkeiten.

In Anbetracht obiger Faktoren soll ein Einblick in das Forschungsprojekt zur Untersuchung von kommunikativen zweisprachigen Praktiken dieser „Noch-Sprechergemeinschaften" kurz dargestellt werden.

\subsection{Kommunikative Handlungsmuster: bilingualer Sprachmodus der Minderheiten}

Zum besseren Verständnis der sprachlichen Handlungsmuster dieser Minderheit, die durch die angeführten Merkmale als dynamisch und äußerst variabel beschrieben werden können, soll deren Hintergrund kurz erläutert werden. Sprachliche Handlungsmuster sind gleichzeitig auch als soziale Praktiken zu betrachten und bilden einen wesentlichen Bestandteil des Alltags- und Weltwissens der Sprecherinnen und -sprecher. Die Kommunikationsfähigkeit wird nämlich nicht allgemein und abstrakt erworben, sondern immer auf konkrete Situationen bezogen, sodass jede Sprachgemeinschaft über spezifische, ihr eigene kommunikative Praktiken verfügt. In der Kommunikationspraxis einer Sprachminderheit, die sich von den übrigen Sprechergruppen und der Mehrheit durch ihre ethnischen und - heute nur noch teils - sprachlichen Charakteristika abhebt, entstehen diese Handlungsmuster anhand von tradierten Konventionen und sozialen Regeln in den von der Sprechergemeinschaft noch gebrauchten Domänen. Abhängig 
vom Alter, dem Beruf und der sozialen Gruppenzugehörigkeit der Sprecherinnen und -sprecher können diese Handlungsmuster mehr oder weniger fest aber auch variabel sein, die sich durch ein häufiges situationsgebundenes Verwenden in bestimmten tradierten Formen, wie Ritualien, Sitten und Bräuche des Jahresverlaufs der Gemeinschaft gefestigt und verbreitet haben. Pragmatisch betrachtet wirken diese kommunikativen Praktiken bei einer Sprachminderheit als Kohäsionsmittel, durch die die Sprecherinnen und Sprecher der Minderheit als Gruppe zusammen gehalten werden können, solange diese Praktiken noch als eigene und typische Handlungsformen der Gemeinschaft empfunden werden. So ist die von den Ungarndeutschen häufig gebrauchte Mischsprache (deutsch-dialektale Sprache durchsetzt von zahlreichen ungarischen Inhaltswörtern) als ein ihr charakteristisches kommunikatives Handlungsmuster zu sehen, das nur gruppenintern und generationsbedingt gebraucht wird. Dies kann an einem Gesprächsausschnitt veranschaulicht werden: Auf eine Frage, gerichtet an eine ältere ungarndeutsche Sprecherin (74 J.), die mit ihrer Tochter und deren Familie in ein neues Haus gezogen ist, antwortet diese:

(2) Des Haus is nai, des is vadonatúj, do muss mr nix dra mache. Hát nem is tudom, wie mir dr'zu kume sain, des war a véletlen, azt hiszem...

(Das Haus ist neu, das ist nagelneu, da muss man nichts dran machen. Ich weiß gar nicht, wie wir dazu gekommen sind, das war ein Zufall, glaube ich...)

Als theoretische Grundlage dieser sprachlichen Handlungsmuster kann folgendes erläutert werden. Die die Sprachminderheit umgebenden narrativen Welten unterscheiden sich zwar im Sprachgebrauch der einzelnen Alters- und Berufsgruppen und Generationen, doch werden diese Narrationen der Welt und der engeren Umgebung in der Landessprache erlebt, durch die Medien, die Mehrheitsgesellschaft vermittelt und geprägt. So erscheint es für Minderheitensprecherinnen und -sprecher als selbstverständlich, dass bestimmte Elemente dieser Narrativen, allen voran gängige Realien, bestimmte Idiome, Sachmodernismen und Fachjargonismen, aber auch Inhaltswörter und Partikeln, in der Landessprache gespeichert werden und in dieser auch abrufbar sind.

Diese sprachlichen Handlungsformen sind wie ein Drehbuch in der erlebten Rede der Narration gespeichert, die in der kognitiv erlebten Sprachrealität, also in ungarischer Sprache, aktiviert werden. Somit bilden auch die in Ungarisch gespeicherten lexikalischen Elemente einen wesentlichen Bestandteil des typischen Alltagswortschatzes der Ungarndeutschen, auch sie gehören in ihr sprachliches Repertoire. Solche typischen Handlungsmuster erlebt man in Dorfgemeischaften selbst in der Umgebung von älteren Sprecherinnen und Sprechern.

Die oben geschilderten Gesprächssituationen gehen einher mit einem spezifischen Sprachmodus. Mehrsprachige Sprecherinnen und -sprecher treffen nämlich in der gegebenen konkreten Situation ihre Sprachwahl, welchen Sprachmodus sie bevorzugen (vgl. Grosjean 1999: 178f.). Das für zweisprachige Sprecherinnen und -sprecher entwickelte Konzept der Sprachmodi bietet die Möglichkeit, dass ein Sprecher, der in einem einsprachigen Sprachmodus spricht, den Kode seiner zweiten Sprache nicht völlig 
ausschaltet, nur zeitweilig inaktiv hält, deshalb kann es auch in einem einsprachigen Modus zu einer Beeinflussung etwa auf der suprasegmentalen oder der lexikalischen Ebene kommen. Gespräche in einem zweisprachigen Modus - wie dies bei Minderheitensprecherinnen und -sprechern der Fall ist - führen zu effizienten und schnellen Sprachwechseln, zu einem häufigen Alternieren der zwei Kodes, weil in diesem Fall die zwei Sprachen gleichzeitig aktiviert werden können. Die die Wahl des Sprachmodus beeinflussenden Faktoren bei den Ungarndeutschen sind die soziolinguistischen Variablen, in erster Linie der Gesprächspartner und seine Sprachkenntnisse, die Kenntnis seiner sprachlichen Präferenzen, sein allgemeines Verhältnis zur Sprache der Interaktion, sein Alter, seine soziale Stellung, das Gesprächsthema, der Ort, die Zeit und der Formalitätsgrad des Gesprächs. So spricht man - falls man die deutsche Sprache beherrscht - mit älteren Ungarndeutschen eher in deutscher oder auch in ungarischer Sprache, da diese Generationen sie gleich gut beherrschen, mit anderen Generationen fast immer ungarisch, da mit ihnen in der Regel Ungarisch gebraucht wird, wohl wissend, dass der Gesprächspartner auch Deutsch versteht.

Typisch sind die in zahlreichen Formen auftauchenden Interaktionen einer asymmetrischen Kommunikation, in denen der lokale deutsche Dialekt der älteren Generation und die ungarische Sprache der jungen Generation aufeinandertreffen. Ein solcher Perspektivenwechsel der Sprecherinnen und Sprecher wird in der neueren Theoriebildung der Mehrsprachigkeit erwähnt, wenn die Sprachen und deren Grammatiken innerhalb einer Interaktion konstitutiv mitbedacht werden (Franceschini 2003: 248). Diese Formen der asymmetrischen Interaktionsmuster zeigen auch, dass mit dem bilingualen Sprachmodus die Grundlagen für eine Theoriebildung der Mehrsprachigkeit auch bei Sprachminderheiten gelegt werden kann. Die Variabilität als inhärentes Merkmal der Sprache zeigt sich konkret in folgenden Kontakterscheinungen: in lexikalischen Entlehnungen (vor allem Inhaltswörter aus dem Ungarischen), im Prozess des Codeswitching, in den Formen der Kalkierung (Entlehnung von morphosynatktischen Strukturmustern), im häufigen Gebrauch von ungarischen Funktionswörtern (Partikeln, textgliedernde Partikeln, Interjektionen, kommunikative Routineformeln, Grußformen), wie in einem Gesprächsausschnitt gezeigt wird, wo eine ältere Sprecherin zu ihrer Nachbarin befragt wird und die Antwort, fast in der Form einer Mischsprache, folgends lautet:

(3) Szerintem, die is valószinüleg net drham, ich hep sie net gsege...(Frau, 72 J.) (Meiner Ansicht nach ist die wahrscheinlich nicht zu Hause, ich habe sie nicht gesehen.)

Die oben angeführten Beispiele von Minderheitensprecherinnen sollten zeigen, wie variabel und dynamisch sich ihre Sprachhandlungsmuster gestalten können und an den bilingualen Sprachmodus sehr gebunden sind. Im folgenden Kapitel sollen diese sprachlichen Eigenheiten mithilfe der sprachbiografischen Methode - ein ebenfalls laufendes Projekt - aus subjektiver Sicht näher beleuchtet werden. 


\subsection{Reflexionen aus sprachbiografischen Interviews mit Ungarndeutschen}

In unserem Sprachbiografie-Projekt, das wir unter älteren Gewährspersonen durchführen, haben wir anhand eines von uns erstellten Fragekatalogs nach subjektiven Reflexionen der Sprecherinnen und Sprecher über jene Lebensphasen oder -episoden gefragt, in denen eine oder mehrere Sprachen oder auch ihr Fehlen (Sprachverlust, -erosion, -mischung und Sprachwechsel s. Knipf-Komlósi 2011) eine für die Gewährspersonen relevante Rolle gespielt haben. Im Weiteren werden Ausschnitte aus Sprachbiografien dargestellt, die als freie narrative Formen des Erzählens einen Einblick in das Verhältnis von Individuum und Sprache(n), in unserem Falle dem Verhältnis zur deutschen Sprache, gewähren (vgl. Franceschini 2001: 112-113). Bei der sprachlich-inhaltlichen Auswertung sprachbiografischer Interviews darf nicht außer Acht gelassen werden, dass die subjektive Rückerinnerung an die eigene Lebensgeschichte (samt sprachlicher Bezüge) kohärent zu sein scheint, wobei sie in Wirklichkeit jedoch fragmentarisch, manchmal sogar widersprüchlich ist (vgl. dazu Arendt 2014: 7-30). Die befragte Person nämlich ergänzt kraft des schöpferischen Aktes des Erinnerns und des strukturierten, reflektierten Nacherzählens die eigenen Reflexionen, die auf diese Weise auch verändert werden können.

Obgleich sprachbiografische Reflexionen als subjektive Daten eingestuft werden, können sie als reliable Daten linguistischer Sprachbiografieforschungen gelten und schriftlichen, mündlichen, ja sogar bildlichen Quellen entnommen werden. Schriftliche Quellen reflektierter Erinnerungen sind z. B. Tagebuchaufzeichnungen, Romane oder Briefe (vgl. König 2017: 200), von Letzteren vornehmlich jene, die für private Zwecke verfasst wurden. Visuell werden erlernte oder erlebte Sprachen samt ihrer affektiven Einschätzung in Form von gezeichneten Sprachportraits (vgl. Krumm/Jenkins 2001) dargestellt, die man im Anschluss an ihre Anfertigung - besonders in Sprachlernsituationen - durch die befragte Person kommentieren lässt, insofern sind bildliche sprachbiografische Daten nicht per se visueller, sondern sekundär auch mündlicher Natur. ${ }^{7}$ Als primär mündliche Quellen gelten spontane subjektive Äußerungen in einem Gespräch, Gruppendiskussionen oder narrative, erzählgenerierende Interviews (Schütze 1983: 285-288).

Aus den sprachbiografischen Interviews, die zur Erhebung der Einstellungen und der Mehrsprachigkeit der Ungarndeutschen durchgeführt wurden, geht hervor, dass ältere Ungarndeutsche (über 60 Jahre, N=126), deren primäre Sozialisation (oder zumindest die ersten Lebensjahre) noch in einem autochthonen deutschen (dialektalen) Umfeld ablief, sich nur bis zu $66 \%$ als zweisprachig (ungarisch-deutsch) betrachten:

7 Bei den bildlichen Sprachportraits können linguistische Laien ihre Sprachen in eine Menschensilhouette durch verschiedene Farben hineinzeichnen, je nach der Stelle, welche Körperteile sie mit den betreffenden Sprachen (z. B. die jeweilige Familiensprache mit dem Herzen, Englisch als Fremdsprache mit dem Kopf) verbinden. Die bildliche Art der Eruierung des sprachlichen Werdegangs wird besonders bei jenen Personen verwendet, die aus kognitiven oder sprachlichen Gründen (z. B. Kinder, Flüchtlinge) über eine eloquente Redeweise in der Sprache der Befragung (noch) nicht verfügen. 
(4) Mia haam nua Schwowisch kräidt tahaam (Wir haben nur Schwäbisch geredet daheim). Mä Aal hod kaa Ungarisch kwust (Meine Großmutter hat kein Ungarisch gewusst). (Frau, $73 \mathrm{~J}$.)

Der Wechsel der Familiensprache - aus der dt. Mundart in die ungarische Standardsprache - erfolgte oft mit dem Eintritt des Kindes in die Schule, in den Kindergarten, Darüber berichtet eine Gewährsperson - diesmal in Standarddeutsch - folgendermaßen:

(5) Mein älterer Bruder wurde zuerst im Dialekt erzogen. Als er in den Kindergarten ging, bat er Mutter, dass sie nur ungarisch reden sollen, weil im Kindergarten alle nur diese Sprache sprechen. Meine Mutter war nicht genug stark und gab nach. (Frau, 68 J.)

Der Sprachwechsel des ältesten schulreif gewordenen Kindes hatte zur Folge, dass die jüngeren Geschwister bereits in eine durchgehend ungarischsprachige familiäre Umgebung hineinwuchsen. Auch die u. U. von außen her forcierte gesellschaftliche Mobilität trug maßgebend dazu bei, dass sich innerhalb der Familie eine sozial bedingte Zweisprachigkeit entwickelte:

(6) Wir sind aus dem Dorf in die Stadt gezogen als ich 6 war, weil uns mit der Schließung der Grundschule gedroht wurde. Ab dann wurde nur auf Ungarisch gesprochen, wenn wir im Dorf waren, dann im Dialekt. (Frau, 68 J.)

Sprecherinnen und Sprecher, die in der Volksschule keinen Deutschunterricht hatten, begegneten dem Hochdeutschen (d. h. der normierten Sprache) relativ spät. Auf die Frage: Wann haben Sie das erste Mal Hochdeutsch gehört? antwortete ein Proband:

(7) Háát, viläächt nochn Forradalom [1956er Revolution]. Ja, ja, tanoch (Ä̈̈m, vielleicht nach der Revolution. Ja, ja, danach). (Mann, 84 J.)

Die Frage, ob die Probanden ihre deutsche Mundart schön finden, erwiderten sie mit gemischten Antworten, zumal hier auch der Aspekt der Nützlichkeit mitschwang:

(8) Jo... is a aunständigi Sproch (Ja... ist eine anständige Sprache). Täis is kemischt (Das ist gemischt). Wenn andere nicht antworten können, dann ist das schwer. (Mann, $75 \mathrm{~J}$.)

Bei der Gegenüberstellung der autochthonen Mundart und der deutschen Hochsprache wurde in der überwiegenden Mehrheit der Fälle die normierte Hochsprache als schöner erachtet:

(9) Mia saan täis kwäint, Schwäbisch, in den Dialekt awe... (Wir sind das gewöhnt, Schwäbisch, in dem Dialekt, aber...) Aber schön... ist die deutsche Sprache. (Mann, 75 J.) 
Trotz der bis Ende der 1960er Jahre dauernden Stigmatisierung des Deutschen in Ungarn waren die Befragten sich dessen bewusst, dass ihre Zweisprachigkeit in der Gegenwart sowohl im beruflichen als auch im privaten Leben eindeutig Vorteile hat:

(10) Ich bin stolz darauf Schwäbin zu sein. Ich kann zwei Sprachen. Wie weit kommst du mit dem Ungarischen? ... [Ich habe mich zurechtgefunden] sogar in Australien. (Frau, $73 \mathrm{~J}$.)

Mit der Mundart sowie mit der hochdeutschen Sprache kamen die Probandinnen und Probanden in der ganzen osteuropäischen Region im Ausland zurecht:

(11) Awe mid de täitsch Sproch kama... kama irgendwohin [überallhin] kuma. Und kama... räin. Sou wi to tanää in Montenegro hama mid ta täitsch Sproche schäi wäädekuma kaine. Oda pä di Serben, oda in Bulgarien... oda pä die Sl... Schl... Slowakien... hama kaine ååf Täätsch räidn. Pä di Rumäine, wo... wos Ainglisch graidt haam, oda, oda Rumänisch, awe trotzdem, awe in Restaurant täätsch bestöönj kaine und verlangen kaine woos

(Aber mit der deutschen Sprache kann man... kann man überallhin kommen. Und kann man... reden. So wie da unten in Montenegro haben wir mit der deutschen Sprache schön weiterkommen können. Oder bei den Serben, oder in Bulgarien... oder bei die [sic] Sl... Schl... [in der] Slowakei... haben wir können auf Deutsch reden. Bei den Rumänen, wo... wo es Englisch geredet haben, oder, oder Rumänisch, aber trotzdem, aber im Restaurant deutsch bestellen können und um etwas bitten können). (Mann, $74 \mathrm{~J}$.)

In den sprachbiografischen Interviews wurde auch danach gefragt, in welcher Lebensphase man wegen des Mundartgebrauchs gebrandmarkt wurde. Diese Lebensphasen fallen auf die ersten Jahrzehnte nach Kriegsende, in denen die deutsche Minderheit wegen der Auferlegung einer Kollektivschuld mit Kriegsverbrechern geleichgestellt wurde. Der öffentliche Gebrauch der deutschen Mundart rief bis in die 1960er Jahre bei der Mehrheitsbevölkerung Unmut und Missfallen hervor:

(12) Meine Mutter hat mir gesagt, als sie noch zur Arbeit gegangen war: in der Straßenbahn kein Wort Schwäbisch. Man hat sie beinahe von der Straßenbahn gestoßen. [Mitte der 1960er Jahre]. Wir haben uns in den Zug gesetzt und [darauf gewartet, dass er losfährt] und dabei ist hin und wieder ein Wort [auf Schwäbisch] gefallen. Daraufhin hat man uns von der Plattform zugerufen: „Ihr seid stinkende Schwaben." Interviewer: Waren dies Männer? Jugendliche. Männer und Frauen. Ich war 14-15 Jahre alt. Wir haben es nicht gewagt, unseren Mund aufzumachen. (Frau, $74 \mathrm{~J}$.) 
Seit den 1970er Jahren, in der (im Vergleich zu den vorangehenden Jahrzehnten) lockereren Atmosphäre der Kádár-Ära trauten sich die Deutschen allmählich ihre Sprache und ihre verdrängte Identität wahrzunehmen. Auf die Frage Wann haben Sie wieder angefangen Schwäbisch zu reden? entgegnete eine Probandin:

(13) Als Schwäbisch nicht mehr verboten war. Dann haben wir angefangen uns zurückzuschwabieren [sic]. In den siebzigern.

Interviewer: Ja, aber wann konkret?

Täis kari tia näd soogn. Awe i klaab, vn tain ist es sche voorkuma. Mia haam uns sche... näd fiacht

(Das kann ich dir nicht sagen. Aber ich glaube, von da an ist es schon vorgekommen. Wir haben uns schon... nicht gefürchtet). (Frau, 74 J.)

Die mit der Ortsmundart verbunden genannten Gefühle zeigen also eine disparate Beurteilung, je nachdem, ob sie verstanden wurde oder nicht: Die Mundartkenntnisse und bei den ProbandInnen, die nach dem Weltkrieg eingeschult wurden, die Hochdeutschkenntnisse brachten sowohl Nachteile als auch Vorteile mit sich. Die Zäsur zwischen öffentlicher Anprangerung, sprachlicher Diskriminierung und gesellschaftlicher Aufwertung des Mundart- und Hochdeutschgebrauchs in Ungarn liegt im letzten Drittel der sechziger, Anfang siebziger Jahre.

\section{ZUSAMMENFASSUNG}

Unsere Besipiele aus den sprachbiografischen Interviews gaben Auskunft darüber, was die befragten Personen im Rückblick und in ihrer Erinnerung über jene Sprachen und Varietäten (deutsche Ortsmundart, normierte deutsche Sprache, ungarische Standardsprache) wissen und denken, die sie als funktionale Erstsprache erworben oder mit denen sie in ihrer Vergangenheit konfrontiert wurden. Anhand der hier angeführten Äußerungen erfährt man explizit und implizit Informationen, Beweggründe über ihr Sprachverhalten heute, über die soziolinguistischen Umstände, die den Spracherwerb dieser Menschen determiniert haben, welche Konzeptualisierungen sie von „Sprache“ entwickelt haben, welche Aspekte ihrer Sprache sie als wichtig erachten und diese in den Interviews zur Sprache bringen, und nicht zuletzt, ob sie sich selbst als Agens oder Patiens im Spracherwerbs- und Sprachgebrauchsprozess sehen. Denn sprachliche Ideologien verschwinden nicht einfach, einerseits schlagen sie sich in ihren/unseren Lebensgeschichten nieder (vgl. Cuonz/Studler 2014), andererseits können sie das Sprachverhalten der Sprecherinnen und Sprecher beeinflussen und dadurch zu Sprach(gebrauchs) veränderungen führen (vgl. Lanstyák 2011: 14).

Die vorgestellten, laufenden Projekte zeigen aktuelle Forschungsfragen und moderne Annäherungen an die Minderheitensprache. Selbstverständlich verlangen die neuen Forschungsfragen auch neue methodische Herangehensweisen, doch sie entschlüsseln auch so manches Rätsel beim Sprachverhalten dieser Sprecherinnen und Sprecher, z. B. die Stigmatisierung und daraus folgend die Verstummung ganzer Generationen, die widersprüchliche Beurteilung der eigenen deutschen Mundartkenntnisse. Hier lässt 
sich auch zeigen, dass objektive Sprachdaten, gekoppelt mit subjektiven Reflexionen der Sprecherinnen und Sprecher, einen besseren Einblick, ein besseres Verständnis für Prozesse des Sprachwechsels, der Sprachwahl, des Sprachabbaus mehrsprachiger Sprecherinnen und Sprecher gewähren können.

\section{Literatur}

ARENDT, Birte (2014) „Qualitative Interviews als interaktive ko-konstruktive Prozesse: Kontextsensitivität in mikroanalytischer Perspektive." In: Ch. Cuonz/R. Studler (Hrsg.), Sprechen über Sprache. Perspektiven und neue Methoden der Spracheinstellungsforschung. Tübingen: Stauffenburg, 7-30.

CUONZ, Christina/Rebekka STUDLER (2014) Sprechen über Sprache. Perspektiven und neue Methoden der Spracheinstellungsforschung. Tübingen: Stauffenburg.

EICHINGER, Ludwig (1997) „Deutsch in weiter Ferne.“ In: G. Stickel (Hrsg.), Varietäten des Deutschen. Berlin: de Gruyter, 155-180.

ERB, Maria (2012) Wenn das Fremde zum Eigenen wird: Korpusbasierte Untersuchungen zu den ungarischen Lehnwörtern der nachtürkischen deutschen Sprachinselmundarten von Ungarn bis 1945. Budapest, Magyarország: ELTE Germanistisches Institut.

ERB, Maria/Elisabeth KNIPF (2000), ,Selbstreflexionen zum Ortsdialekt in den deutschen Ortschaften im Ofner Bergland." In: A. Greule/F. Scheuerer/L. Zehetner (Hrsg.), Vom Sturz der Diphtonge. Beiträge zur 7. Arbeitstagung für bayerisch-österreichische Dialektologie in Regensburg. Tübingen: Narr, 217-229.

FRANCESCHINI, Rita (2001) „Sprachbiographien randständiger Sprecher.“ In: R. Franceschini (Hrsg.), Biographie und Interkulturalität. Diskurs und Lebenspraxis. Tübingen: Stauffenburg, 111-125.

FRANCESCHINI, Rita (2003) „Modellbildung über die Mehrsprachigkeit hinaus: für eine Linguistik der Potentialität.“ In: L. Mondada/S. Pekarek Doehler (Hrsg.), Plurilinguisme, Mehrsprachigkeit, Plurilingualism. Tübingen/Basel: A. Francke, 247-259.

GROSJEAN, Francois (1999) „The bilingual's language modes.“ In: J. L. Nicol/T. Langendoen (Hrsg.). Language Processing in the Bilingual. Oxford: Blackwell.

KNIPF, Elisabeth/Márta MÜLLER (2019) „Fördermaßnahmen zum Erhalt der deutschen Sprache in Ungarn. “In: U. Ammon/G. Schmidt (Hrsg.), Förderung der deutschen Sprache weltweit: Vorschläge, Ansätze und Konzepte. Berlin/Boston: De Gruyter, 483-500.

KNIPF-KOMLÓSI, Elisabeth (2011) Wandel im Wortschatz der Minderheitensprache. Am Beispiel des Deutschen in Ungarn. Stuttgart: Franz Steiner Verlag.

KÖNIG, Katharina (2017) „Das sprachbiographische Interview als Interaktion. Eine gesprächsanalytische Perspektive auf ein Forschungsinstrument.“ In: C. Jürgens/I. Schröder (Hrsg.), Sprachliche Variation in autobiographischen Interviews. Theoretische und methodische Zugänge. Frankfurt a. Main (etc.): Peter Lang, 199-224.

KRUMM, Hans Jürgen /Eva-Maria Jenkins (Hrsg.) (2001) Kinder und ihre Sprachen: lebendige Mehrsprachigkeit. Wien: Wienerverlagswerkstatt. 
LANSTYÁK, István (2011) „A nyelvi ideológiák néhány általános kérdéséről.“ [Über einige allgemeine Fragen von sprachlichen Ideologien.] In: K. Misad/Z. Csehy (Hrsg.), Nova Posoniensia. A pozsonyi magyar tanszék évkönyve. Pozsony: Szenczi Molnár Albert Egyesület-Kalligram Kiadó, 13-57.

MANHERZ, Karl (1986) Deutsche Mundarten im Pilisch-Gebirge. Ungarndeutsche Studien 3. Budapest: Tankönyvkiadó.

MÁRKUS, Éva (2014) Die deutsche Mundart von Deutschpilsen/Nagybörzsöny. Wien: Praesens.

MÜLLER, Márta (2011) Lexikalisch-semantische Merkmale der Handwerk-Fachwortschätze in Werischwar/Pilisvörösvár. Budapest: ELTE Germanistisches Institut.

SCHÜTZE, Fritz (1983) „Biographieforschung und narratives Interview.“ Neue Praxis, 13(3), 283-293. https://nbn-resolving.org/urn:nbn:de:0168-ssoar-53147 (02.02.2020).

WILD, Katharina (2003) Zur komplexen Analyse der 'Fuldaer' deutschen Mundarten Südungarns. Budapest: ELTE Germanistisches Institut.

\section{Zusammenfassung \\ AKTUELLE FRAGEN DER DEUTSCHEN MINDERHEITENFORSCHUNG IN UNGARN}

Nach der Klärung der soziolinguistischen Merkmale der Sprachkonstellationen von ungarndeutschen Minderheitensprecherinnen und -sprechern wird im Beitrag der Versuch unternommen, durch Belege aus sprachbiografischen Interviews zu untermauern, welches Potenzial die Auseinandersetzung mit den Erfahrungen, Einstellungen, Verhaltens- und Handlungsmustern von Minderheitensprechern des Deutschen in Ungarn birgt, die die Sprecherinnen und Sprecher im Laufe ihres Lebens entwickelt haben.

Es wird darauf eingegangen, welche variablen kommunikativen Praktiken für Minderheitensprecher charakteristisch sind, wie sich ihr sprachliches Umfeld im Laufe ihrer Biografie gestaltete, wie sie diese Lebensphasen sprachlich erlebt haben und heute bewerten, bzw. welchen Nutzen sie ihrer Zweisprachigkeit beimessen.

Schlüsselwörter: Deutsch als Minderheitensprache in Ungarn, Dialektsprecher, kommunikative Handlungen, Einstellungen zum Deutschen, sprachbiografische Interviews

\section{Abstract \\ CURRENT QUESTIONS OF THE RESEARCH ON THE GERMAN MINORITY IN HUNGARY}

After a presentation of the sociolinguistic conditions of the German speakers in Hungary, the paper discusses the usefulness of interviews through which language biographies can be constructed on the basis of the speakers' experiences and practices as 
well as of their attitudes, patterns of behaviour and activities. Examples are provided from interviews with some German speakers from Hungary of different generations. In particular, we try to find out which specific communication skills and practices are characteristic of the speakers of German as a minority language, what memories they have of their past linguistic circumstances, how they perceive their linguistic development and what value they attribute to bilingualism.

Keywords: German as a minority language, dialect speakers, communicative activities, attitudes to the German language, language biographies

\section{Povzetek \\ AKTUALNA VPRAŠANJA RAZISKOVANJA NEMŠKIH MANJŠIN NA MADŽARSKEM}

V prispevku so najprej opisane sociolingvistične značilnosti jezikovnih konstelacij pri govorcih madžarskonemške manjšine, temu pa sledi opis raziskovalnega potenciala jezikovnobiografskih intervjujev, kar je v prispevku podprto z zgledi iz raziskovalnega gradiva. Takšno raziskovanje namreč prinaša soočanje $\mathrm{z}$ izkušnjami, mnenji ter vzorci jezikovnega vedenja in delovanja nemških govorcev, ti pa so jih kot pripadniki nemške manjšine na Madžarskem razvili v različnih obdobjih svojega življenja. V prispevku je prikazano, katere spreminjajoče se komunikacijske prakse so značilne za manjšinske govorce, kako se je - z biografskega vidika - spreminjalo jezikovno okolje, kako so doživljali življenjska obdobja z ozirom na jezik in kako jih ocenjujejo sedaj oz. kakšno uporabno vrednost pripisujejo svoji dvojezičnosti.

Ključne besede: nemščina kot manjšinski jezik na Madžarskem, narečni govorec, sporočanjska dejanja, odnos do nemščine, jezikovnobiogafski intervjuji 